\title{
RESULTS OF TREATMENT WITH SALVARSAN IN LATE CONGENITAL SYPHILIS *
}

\author{
GEORGE S. STRATHY, M.D., C.M., M.R.C.S., L.R.C.P. \\ Assistant Pathologist Hospital for Sick Children, \\ AND \\ GEORGE A. CAMPBELL, B.A., M.B. \\ House Physician Hospital for Sick Children, \\ TORONTO
}

During the past year we have treated with salvarsan eighteen children who were suffering from congenital syphilis. Our results as regards the Wassermann reaction were so surprising to ourselves that we searched the literature to ascertain the results of others and found so few reports controlled by the laboratory test that we think it worth while to report our cases.

Although the literature on salvarsan treatment is now enormous, very little work has been reported on its use in congenital syphilis. Among British writers, McDonagh ${ }^{1}$ reports a case, aged 15 years, with disappearance of clinical signs, but with the Wassermann reaction positive after one dose. Sequeira ${ }^{2}$ reports one case, also aged 15 years, in which he gave four doses of salvarsan intramuscularly; the remainder of his congenital cases were infants. In his cases the symptoms disappeared, but the reaction remained positive. He seldom gave more than one dose. Marshall ${ }^{3}$ denounces salvarsan. Eddington and Browning ${ }^{4}$ had a patient aged 16 years with a syphilitic knee. After two doses (0.2 and $0.27 \mathrm{gm}$.) of salvarsan the reaction became negative. Bunch ${ }^{5}$ obtained a negative reaction in an infant of 8 weeks after two administrationg, but an infant of 14 weeks was still positive after two doses.

In American literature the reports are merely reports of a few cases, and the effect of the Wassermann reaction is seldom mentioned. De Buys ${ }^{6}$ reports three cases. He advises treatment through the mother's milk, and says salvarsan should only be used as an adjunct to mercury and potassium iodid.

The most thorough reports are by German and Austrian writers, but their reports are mostly on the treatment of nurslings through the

* Submitted for publication May, 1913.

1. MeDonagh, J. E. R.: Brit. Jour. Child. Dis., October, 1910.

2. Sequeira: Brit. Jour. Child. Dis., February, 1911.

3. Marshall: Brit. Jour. Child. Dis., March, 1911.

4. Eddington and Browning: Glasgow Med. Jour., October, 1911.

5. Bunch: Brit. Jour. Child. Dis., April, 1912.

6. DeBuys: Amer. Jour. Dis. Child., January, 1913.

7. Herxheimer and Reinke: Deutsch. med. Wchnschr., September, 1910. 
mother's milk. Herxheimer and Reinke ${ }^{7}$ report two fatal cases, both infants aged 2 months, and both dying four days after the administration. At autopsy arsenic was found in all the organs, but no treponema, except in the lungs, where they were agglutinated and markedly degenerated. Welde $^{8}$ treated twenty-eight patients, partly by subcutaneous and intramuscular injection, but his later cases by the intravenous method. His average dose was $0.1 \mathrm{gm}$., but most of his cases were in infants and received only one administration. The symptoms disappeared, but in only one case did the Wassermann become negative, and then it became positive again. No bad effects were observed.

We have treated eighteen patients; only three of these were under 5 years of age; most of them were suffering from bone lesions or interstitial keratitis. At the outset of our work, like most workers at that time, we believed that one or two doses would cure nearly all cases of syphilis, but by testing the Wassermann reaction after each administration we found it positive, and as the clinical symptoms did not entirely disappear in several cases we repeated the administration. Our method of procedure at first was to give salvarsan about once a month and test the reaction a month later. Later we gave a dose every week or every second week and tested the reaction usually between forty-eight and seventy-two hours following. We did not consider the reaction as finally negative unless the blood was tested between forty-eight and seventy-two hours after an administration, and we agree with McDonagh as to the value of a provocative dose, both in congenital and acquired syphilis. In ninety-nine administrations we used the intravenous method, except in one case (E. M.), who being mentally deficient, resisted, and we had to resort to intramuscular administration. To one who is experienced in giving intravenous injections the small veins of children do not offer a great obstacle. In most cases we used the median basilic or median cephalic veins, but occasionally we had to use the external jugular, placing the child in position advised by Wollstein and Morgan ${ }^{9}$ for blood-culture work. In only one case, a marantic infant of 6 months, did we fail to enter a vein.

The amount given varied according to the weight of the child. Taking 150 pounds as the average male adult weight and $0.6 \mathrm{gm}$. salrarsan and $0.9 \mathrm{gm}$. neosalvarsan as average doses for that weight, we dissolved the salvarsan in 300 c.c. of distilled water and the neosalvarsan in 150 c.c. Of the latter we gave 1 c.c. and of the former 2 c.c. for each pound of weight of the patient.

8. Welde: München. med. Wehnsehr., April, 1912.

9. Wallstein and Morgan: AMER. Jour. Dis. CHrLd, October, 1912. 
THE CLINICAL RESULTS

The administration of salvarsan was in all cases followed by improvement in the physical signs. In no case did the symptoms resist repeated doses, although in one case (C. B.) there was a recurrence of symptoms one month after treatment had been discontinued, the patient having received nine doses. Three patients were babies suffering from wasting and rashes. All three were cured as regards symptoms. Seven patients suffered from interstitial keratitis. The inflammation of the cornea subsided in all cases, leaving, of course, scars, but the subsidence was at least as rapid as with mercury. C. B., aged 10 years, improved rapidly after the fourth dose and also showed marked improvement in his general health, but his symptoms recurred as mentioned above, and he again failed in general condition. Under mercury he improved again. He is the only patient in whom the treatment did not prove satisfactory. He also showed syphilitic arthritis of both knees, which was entirely cured, leaving no deformity. Seven of the patients were cases of bone syphilis, three affecting the nasal bones and causing severe ozena. The symptoms in all except the nasal cases subsided rapidly; one (R. D.) relapsed two months after her first dose, but her symptoms have entirely disappeared after four more doses. The nasal cases have all improved, but are slower and are still under treatment.

One patient had no symptoms except poor bodily development and Hutchinson's teeth. His Wassermann reaction was doubtful, that of his mother positive. He was only given one dose, after which his reaction was negative.

With the exception of R. D., all the children were under-sized and poorly nourished. The improvement in their general health was marked, resembling the improvement after operations in severe cases of enlarged tonsils and adenoids.

The only untoward results noticed were that three bone cases developed mild interstitial keratitis after treatment was begun, and one patient developed it in his right eye while undergoing treatment for the left. In no case were there severe symptoms following the administration, and after the neosalvarsan seldom any nausea at all. Some of them had romiting for twelve to twenty hours after the administration of the original salvarsan.

\section{THE EFFECTS ON THE WASSERMANN REACTION}

As will be seen in the table, only five cases gave a negative reaction following treatment.

A. S., aged 6 months, was positive before treatment, received one dose of neosalvarsan and then for four months mercury by inunction. She then reacted negatively.

A. W., aged 17 months, reacted negatively after three doses, but his blood was not tested previously. His mother's blood was positive. 
H. P., aged 12, reacted negatively after his eighth dose of neosalvarsan.

T. C., aged 12, received three doses of salvarsan and then three doses of neosalvarsan, after which he reacted negatively.

H. F., referred to above, reacted doubtfully before and negatively after one dose of neosalvarsan.

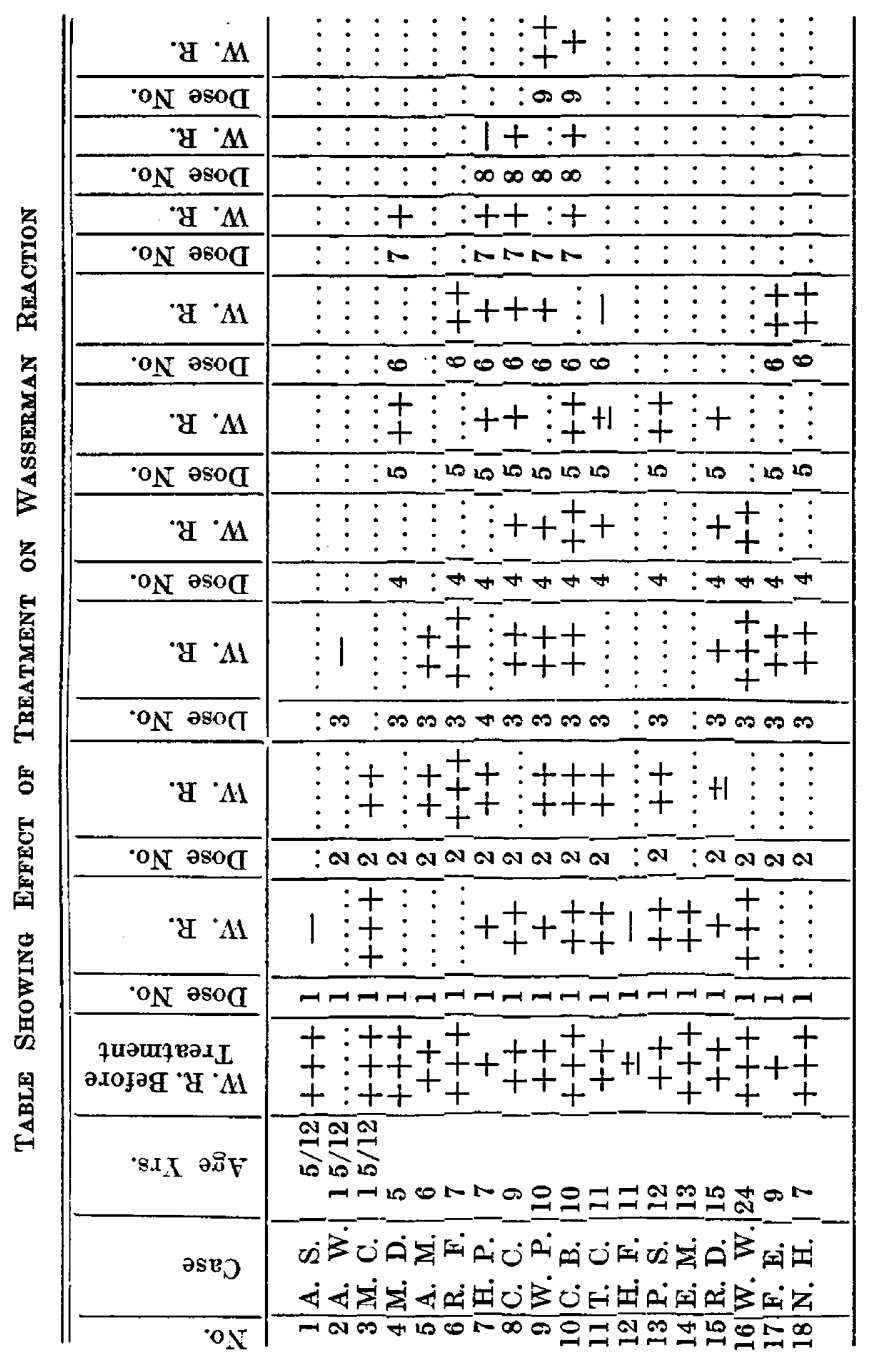

The results of treatment on the Wassermann reaction can hardly be considered satisfactory, except as they compare favorably with the results obtained by mercurial treatment.

Gummata, periostitis and ulcers disappeared rapidly. Keratitis, which was present in eight cases, healed, we believe, more rapidly than under mixed treatment, and the resulting scars were therefore less. The results 
were better than those obtained with mercury, and in about half of the cases followed treatment by mercury where the latter had been of little benefit.

Latterly we have used larger doses, and in future we shall use more intensive treatment, as large doses as the patient can stand and at shorter than seven-day intervals.

Nearly all the patients were treated in the out-patient department of the hospital and were sent home in a few minutes.

\section{CONCLUSIONS}

1. All our own cases improved clinically under salvarsan treatment.

2. The intensity of the Wassermann reaction diminished steadily with frequently repeated full doses, but in nine patients over 4 years, who received four doses or more, only two became negative, and that after eight and nine doses.

3. The younger the child, the more quickly does the Wassermann reaction become negative.

4. We have not found the administration of salvarsan by intravenous injection in children to produce any bad effects. 